\title{
LHCb Silicon Tracker Performance Studies
}

\author{
S. Köstner, B. Adeva, M. Agari, C. Bauer, D. Baumeister, A. Bay, R. P. Bernhard, R. Bernet, J. Blouw, \\ B. Carron, Y. Ermoline, D. Esperante, R. Frei, J. Gassner, W. Hofmann, S. Jimenez-Otero, K. T. Knöpfle, \\ F. Lehner, S. Löchner, C. Lois, M. Needham, A. Perron, V. Pugatch, M. Schmelling, B. Schwingenheuer, \\ M. Siegler, O. Steinkamp, U. Straumann, M. T. Tran, P. Vazques, A. Vollhardt, D. Volyanski, and H. Voss
}

\begin{abstract}
LHCb is one of the experiments for the Large Hadron Collider at CERN and is dedicated to B-physics and CP-violation measurements. To exploit its physics potential good tracking performance with high efficiency in a high particle density environment close to the beam pipe is required. Silicon strip detectors with large read-out pitch and long strips will be used for the $\mathrm{LHCb}$ Inner Tracker behind the magnet and the Trigger Tracker station in front of the magnet. We report here about the design of the Silicon Tracker, test beam results and the electrical tests foreseen during production.
\end{abstract}

Index Terms-Large read-out pitch, long read-out strips, siliconmicrostrip detector, silicon tracker, tracking detector.

\section{INTRODUCTION}

$\mathbf{T}$ HE LHCb detector [1], currently under construction at the Large Hadron Collider at CERN, is designed to study the physics of b-flavoured hadrons. The experiment will operate at a luminosity of $2 \times 10^{32} \mathrm{~cm}^{-2} \mathrm{~s}^{-1}$ resulting in an expected production of $10^{12} \mathrm{bb}$-pairs per year.

Exploiting the angular distribution of the $\mathrm{b} \overline{\mathrm{b}}$-pairs, which are produced predominantly at low polar angles, $\mathrm{LHCb}$ is designed as a single arm forward spectrometer with an acceptance of $15 \mathrm{mrad}$ to $300 \mathrm{mrad}$ in the bending plane of the spectrometer magnet. A silicon-microstrip vertex detector [2] surrounding the interaction region and the Trigger Tracker (TT-station) [1] located in the fringe fields in front of the $4 \mathrm{Tm}$ dipole magnet together with the tracking stations T1-T3 downstream the magnet are used to reconstruct charged particle trajectories. To provide a higher granularity in the high particle density region near the beam-pipe, the tracking stations T1-T3 are split into the Inner Tracker [3], using silicon-microstrip detectors and the Outer

Manuscript received November 13, 2004; revised May 14, 2006

S. Köstner is with the CERN, 1211 Geneva 23, Switzerland and also with the Physik-Institut der Universität Zürich, Winterthurerstr. 190, 8057 Zürich, Switzerland (e-mail: stefan.koestner@cern.ch).

B. Adeva, D. Esperante, C. Lois, and P. Vazques are with the Universidad de Santiago de Compostela, Campus Universitario Sur, 15706 Santiago de Compostela, Spain.

M. Agari, C. Bauer, D. Baumeister, J. Blouw, W. Hofmann, K. T. Knöpfle, S. Löchner, M. Schmelling, and B. Schwingenheuer are with the Max Plank Institut für Kernphysik, Saupfercheckweg 1, 69117 Heidelberg, Germany.

A. Bay, B. Carron, R. Frei, S. Jimenez-Otero, A. Perron, M. T. Tran, and H.

Voss are with the LPHE, EPFL Lausanne, Bât. des Sciences Physiques, 1015 Dorigny, Switzerland.

R. P. Bernhard, R. Bernet, Y. Ermoline, J. Gassner, F. Lehner, M. Needham, M. Siegler, O. Steinkamp, U. Straumann, A. Volhardt, and D. Volyanski are with the Physik-Institut der Universität Zürich, Winterthurerstr. 190, 8057 Zürich, Switzerland.

V. Pugatch is with the Ukranian Academy of Science, UA-252 143, Kiev 143, Ukraine.

Digital Object Identifier 10.1109/TNS.2006.878578

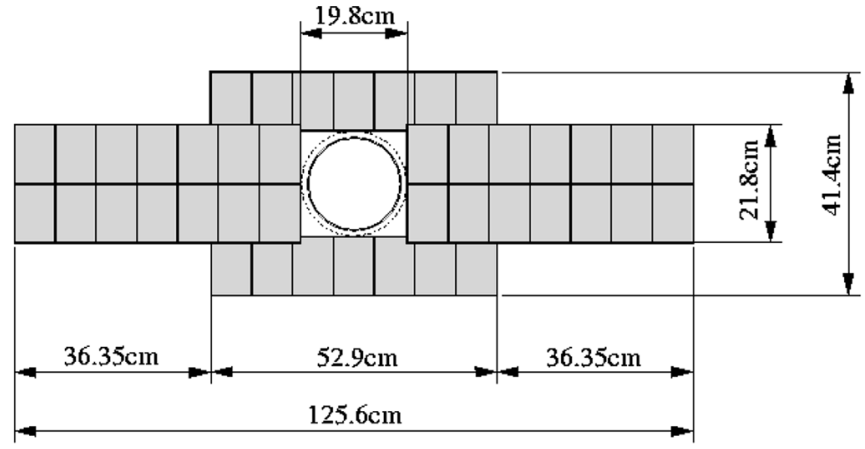

Fig. 1. The layout of a detection layer in an Inner Tracker station, consisting of four individual detector boxes.

Tracker [4] built from straw-tubes. Although the Inner Tracker covers only $1.3 \%$ of the total active area of the tracking stations, about $20 \%$ of the reconstructed charged tracks pass through its acceptance. The occupancy is expected to be less than $2 \%$. The TT-station covers the full acceptance of the experiment and is used to measure the transverse momentum of large impact parameter tracks in the Level-1 trigger. The operation temperatures of the TT-station and the Inner Tracker are foreseen to be $5^{\circ} \mathrm{C}$ in order to reduce the laeakage currents caused by radiation damage after several years of operation. Fluences of up to $5.5 \times 10^{13} \mathrm{~cm}^{-2}$ of $1 \mathrm{MeV}$ neutron equivalent are expected at the centre of the TT-station in 10 years of operation.

\section{DESIGN OF THE SILICON TRACKER}

The Silicon Tracker project comprises the Inner Tracker and the Trigger Tracker. Simulation studies have shown that the momentum resolution in LHCb is dominated by multiple scattering up to about $80 \mathrm{GeV} / \mathrm{c}$. This results in a spatial resolution requirement which is met by silicon micro-strip detectors with a read-out pitch of the order of $200 \mu \mathrm{m}$. Large read-out pitch and long read-out strips, adapted to the expected hit occupancies, are used throughout the Silicon Tracker in order to reduce the number of read-out channels and hence the costs. The Silicon Tracker covers a total area of $12.2 \mathrm{~m}^{2}$ with 270000 read-out channels.

Each Inner Tracker station [3] consists of four individual detector boxes which are arranged around the beam pipe as shown in Fig. 1. The side boxes contain Si-ladders that are $22 \mathrm{~cm}$ long and are built out of two sensors. The detector boxes above and below the beam-pipe employ single-sensor ladders. Each detector box houses $28 \mathrm{Si}$-ladders in four detection layers, with the two outer layers having vertical read-out strips and the two 


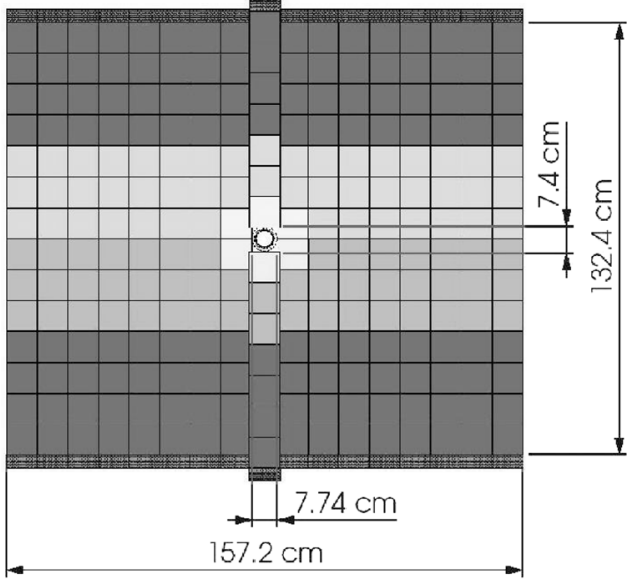

Fig. 2. The layout of one layer in the TT-station (TTb). The entire LHCb angular acceptance is covered by silicon strip detectors. Short read-out sectors are used in the centre around the beam pipe, longer read-out sectors of up to four sensors elsewhere.

inner layers placed with a $\pm 5^{\circ}$ stereo angle. The boxes provide electrical and thermal insulation.

The TT-station consists of four layers arranged in two half stations, TTa and TTb, separated by $30 \mathrm{~cm}$ along the beam axis and enclosed in a single box providing electrical and thermal shielding. Perpendicular to the beam axis, the box is split in order to retract the two halves to allow access to the beam-pipe for the bake-out procedure. As in the Inner Tracker, the first and the fourth layer have vertical read-out strips, while the inner two layers have a stereo angle of $\pm 5^{\circ}$.

The sensors are arranged in read-out sectors of one- and two sensors in the centre around the beam pipe, as indicated in Fig. 2. Read-out sectors consisting of three sensors bonded together are used near the centre of the bending plane and four sensors, i.e., $38 \mathrm{~cm}$ long strips, in the upper and lower area of the detector. The read-out hybrids are located at the edge of the detector, outside of the acceptance. The inner sectors are connected to the read-out hybrids via $40 \mathrm{~cm}$ long Kapton interconnect cables. A cable of $58 \mathrm{~cm}$ length connects to the single sensor near the beam pipe. This design results in load capacitances of up to 57 $\mathrm{pF}$ for the pre-amplifier.

\section{Test BEAm Results}

Several prototype modules with different sensor thicknesses, strip lengths and implant width over pitch ratios were built and then tested in a $120 \mathrm{GeV} / \mathrm{c} \pi^{-}$beam at CERN [9]. One-, twoand three-sensor long ladders were built from multigeometry sensors [5] with a thickness of $320 \mu \mathrm{m}$. In addition three-sensor ladders were built from GLAST [6] and CMS [7] sensors, with thicknesses of $410 \mu \mathrm{m}$ and $500 \mu \mathrm{m}$ respectively. The sensors were chosen as their geometries and thicknesses are close to these expected to be required for the optimal design of the longer TT-ladders. The geometries of the various detectors are summarized in Table I. Their technology is based on single-sided $\mathrm{AC}$ coupled $\mathrm{p}^{+} \mathrm{n}$ silicon strip sensors as chosen for the Silicon Tracker. The read-out is provided via the Beetle preamplifier chip [8][15]. The Beetle is a 128 channel custom made analog
TABLE I

Prototype Sensors Properties. LHCb A to D Labels the Different REGIONS ON THE MULTI-GEOMETRY SENSORS (LHCB). FOR THE LADDER WITH THE KAPTON CABLE THE TOTAL LENGTH IS QUOTED

\begin{tabular}{|c|c|c|c|c|c|c|}
\hline Type & $\begin{array}{c}\text { thickness } \\
{[\mu \mathrm{m}]}\end{array}$ & $\begin{array}{c}\text { pitch } \\
{[\mu \mathrm{m}]}\end{array}$ & $\begin{array}{c}\text { width } \\
{[\mu \mathrm{m}]}\end{array}$ & w/p & $\begin{array}{c}\text { length } \\
{[\mathrm{cm}]}\end{array}$ & $\begin{array}{c}\text { tot.Cap. } \\
{[\mathrm{pF}]}\end{array}$ \\
\hline LHCb A & 320 & 198 & 50 & 0.252 & 10.8 & 17.8 \\
\hline LHCb B & 320 & 198 & 60 & 0.303 & 10.8 & 17.9 \\
\hline LHCb C & 320 & 198 & 70 & 0.354 & 10.8 & 19.5 \\
\hline LHCb D & 320 & 240 & 70 & 0.295 & 10.8 & 18.8 \\
\hline GLAST & 410 & 228 & 56 & 0.246 & 26.3 & 43.3 \\
\hline CMS3 & 500 & 183 & 46 & 0.251 & 28.9 & 41.6 \\
\hline CMS3+flex & 500 & 183 & 46 & 0.251 & 68.9 & 57 \\
\hline
\end{tabular}

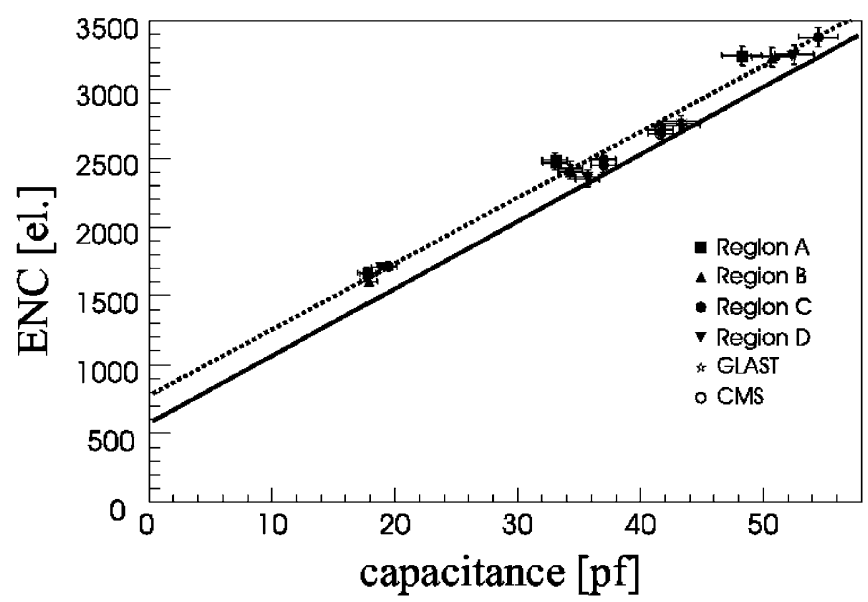

Fig. 3. The equivalent noise charge obtained with the Beetle read-out chip as a function of its input load capacitance. Regions A-D correspond to different implant widths and strip pitch on LHCb Inner Tracker prototype sensors. Other measurements were taken with modules built from CMS and GLAST sensors. The dashed line is the best fit to the test-beam data while the solid line is the expectation from laboratory measurements.

read-out chip using commercial $0.25 \mu \mathrm{m}$ CMOS technology with a radiation hard design. It operates at $40 \mathrm{MHz}$ and saves events into a pipeline with a maximum latency of 160 clock cycles. An important feature of the Beetle is that the shaping time can be varied via an internal programmable register $\left(V_{f s}\right)$ which changes the feedback resistance in the shaper. Thus it allows to optimize signal shape versus noise performance for different ladder geometries with differing capacitive loads. The Beetle version 1.2 is used in these measurements. As no changes in the frontend were implemented in version 1.3, which will be used in the final experiment, the performance concerning the $\mathrm{S} / \mathrm{N}$ ratio is expected to be similar, as verified in a later testbeam [13]. The ladders were tested under room temperature. The read-out electronics were attached to copper cooling blocks in order to remove the produced heat. The Beetle chip was clocked at 25 ns. The studies are based on single track events, which were the dominating events at the provided beam intensities.

Based on the expected charge generated in the sensors, the equivalent noise charge for different strip capacitances was deduced from the observed $\mathrm{S} / \mathrm{N}$ values. The results are shown in Fig. 3, together with the expected noise from laboratory measurements, where load capacitances were connected to the input of the Beetle chip on a test-bench. Good agreement is found for the dependence of the noise on the load capacitance, with a small offset indicating an additional constant noise source in the 


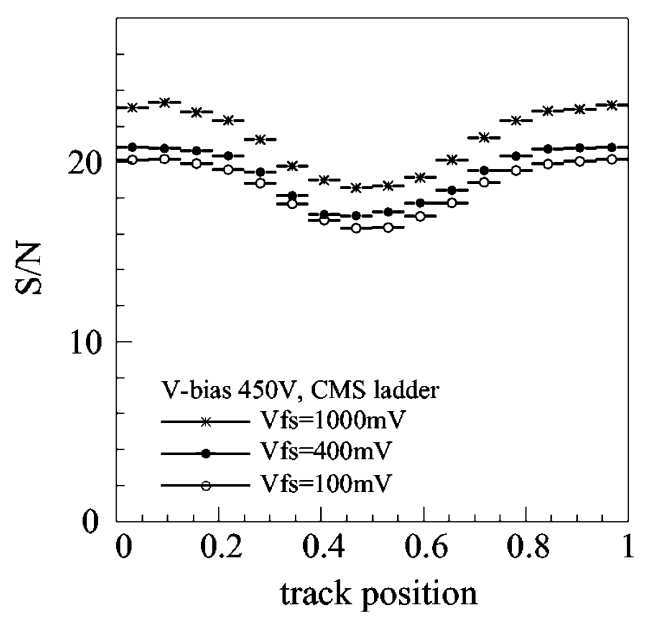

Fig. 4. The $\mathrm{S} / \mathrm{N}$ for different shaping times, depending on the relative positions of the incident particle with respect to the read-out strips. Positions 0 and 1 correspond to the centre of adjacent read-out strips.

test-beam setup. The observed dependence can be used to estimate the expected $\mathrm{S} / \mathrm{N}$ for modules with increased load capacitances due to Kapton interconnect cables or additional sensors. The parameterization is fitted as $\mathrm{ENC}=542+49 \times \mathrm{C}$, where $\mathrm{C}$ denotes the total capacitance (the sum of backplane capacitance and inter-strip capacitance).

Fig. 4 shows measurements of the $\mathrm{S} / \mathrm{N}$ ratio for different relative positions of the incident particle with respect to the read-out strips, for a module built out of three CMS-OB2 sensors. The decrease of the $\mathrm{S} / \mathrm{N}$ ratio in between the strips is due to a charge loss that was already observed in previous measurements [3]. The final detector design requires the $\mathrm{S} / \mathrm{N}$ ratio to be high enough also in between two read-out strips. To obtain full cluster finding efficiency at a reasonable noise-rate, a $\mathrm{S} / \mathrm{N}$ ratio of at least 10 is required. Adding a safety margin, the $\mathrm{S} / \mathrm{N}$ value should be higher than 12 over the whole detector.

Since several different sensor types have been tested it is possible to investigate the dependence of the charge loss in the inter-strip region on the design geometry [9]. First, tracks that pass within \pm 0.1 in pitch units around a strip center are selected. The ADC counts on the three strips closest to the track are then summed and the resulting distribution fitted with a Landau convolved with a Gaussian. The MPV of the Landau from the fit is then taken as the size of the on-strip charge, $C_{\mathrm{on}}$. Tracks that pass within \pm 0.1 around the middle of two read-out strips are selected and the four strips closest to the track summed. Again the resulting distribution is fitted with a Landau convolved with a Gaussian. The MPV of the fitted Landau of this distribution is the size of the mid-strip charge, $C_{\text {mid }}$. The relative dip, $r_{d}$ is then defined as:

$$
r_{d}=C_{\mathrm{mid}} / C_{\mathrm{on}}
$$

In Fig. 5, $r_{d}$ is plotted against $x_{d}=(p-w) / t$ where $p$ is the pitch, $w$ the implant width and $t$ the detector thickness for the sensor geometries that were tested. It can be seen that $r_{d}$ depends approximately linearly on $x_{d}$ in the range investigated. A linear fit gives:

$$
r_{d}=1.06-0.66 \times x_{d} \text {. }
$$

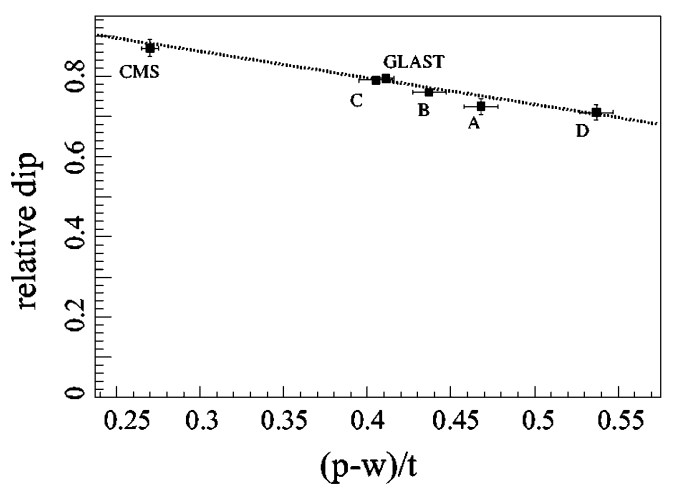

Fig. 5. Relative dip as a function of $x_{d}=(p-w) / t$. In the investigated parameter space the relative dip can be approximated by a linear fit as a function of pitch $(\mathrm{p})$ width $(\mathrm{w})$ and thickness $(\mathrm{t})$.

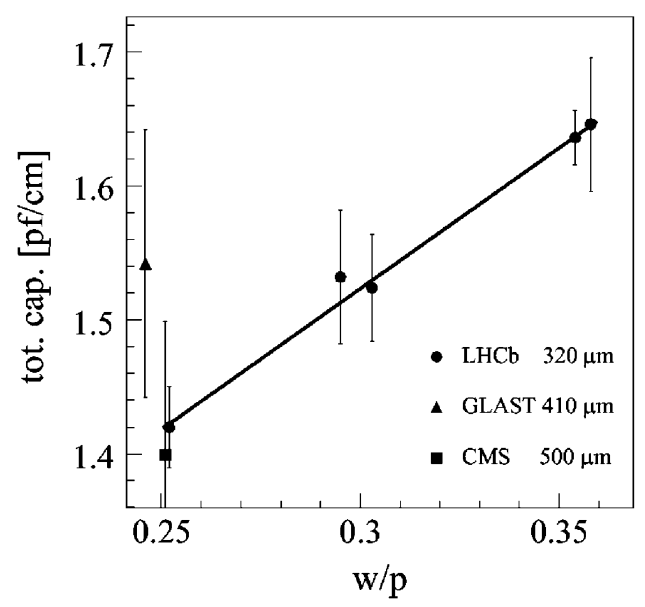

Fig. 6. Dependence of the total capacitance on the ratio w/p (strip width over read-out pitch). The figure shows a linear fit as a function of w/p for the total capacitance.

This relationship means that the relative size of the dip can be reduced either by increasing w/p or by increasing the sensor thickness. A similar procedure can also be carried out by only considering the charge on the closest strip to the track in the on-strip case and the two strips closest to the track in the midstrip case. Again an approximately linear dependence is found with:

$$
r_{d}=1.09-0.60 \times x_{d}
$$

Using the capacitance measurements from [10] and plotting them as a function of the ratio of width over pitch, w/p, we confirm a linear dependence as already observed in [11]. The fit is shown in Fig. 6. As in [11] the total capacitance appears to be independent of the thickness of the detector. The fit was obtained from the data points of the multigeometry sensors only and resulted in an offset of $0.89 \mathrm{pF} / \mathrm{cm}$ and a slope of $2.1 \mathrm{pF} / \mathrm{cm}$. The offset is comparable with the results quoted in [11] whereas the derived slope is higher. The GLAST ladder, however, does not fit into the linear behaviour. A systematic difference is expected for this ladder, because the bias resistors which are an order of magnitude higher for the GLAST ladder are expected to 

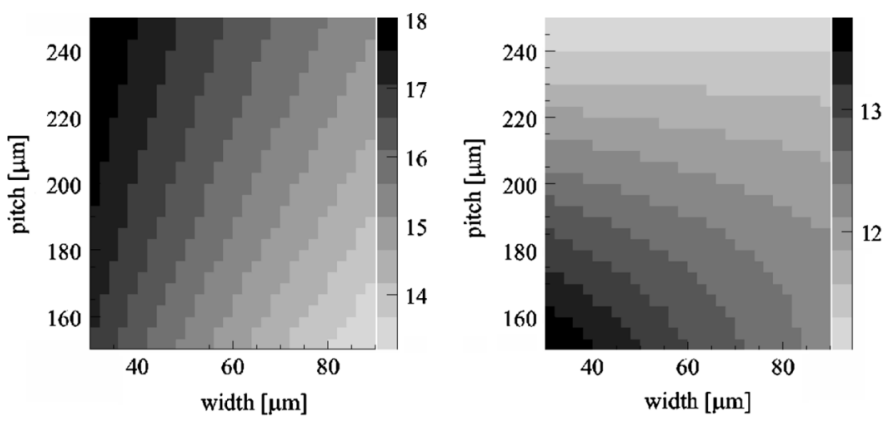

Fig. 7. S/N ratio for the on-strip region (on the left) and the inter-strip region (on the right) as a function of strip width and read-out pitch. The $\mathrm{S} / \mathrm{N}$ for the on-strip data decreases from the top left corner down to the right bottom corner. For the inter-strip data the $\mathrm{S} / \mathrm{N}$ increases from the bottom left corner up to the top right corner.

affect the frequency dependent measurements carried out with a LCR-meter [10].

How these parameterizations can be used to estimate the $\mathrm{S} / \mathrm{N}$ value is shown in [12] for a $320 \mu \mathrm{m}$ thick sensor of $10.8 \mathrm{~cm}$ length. The injected signal per track is expected to be 24730 electrons [9]. The internal noise from the Beetle read-out chip can be considered as dominant noise source, which is proportional to the total strip capacitance, as shown above. For the total strip capacitance the parameterization can be inserted and multiplied with a strip length of $10.8 \mathrm{~cm}$ for a single sensor LHCb ladder. Dividing the injected charge by the equivalent noise charge gives the $\mathrm{S} / \mathrm{N}$ ratio for tracks passing close to the read-out strip (on-strip). The dependence on pitch and width for the $\mathrm{S} / \mathrm{N}$ ratio of the on-strip tracks is shown in Fig. 7. For a pitch of $200 \mu \mathrm{m}$ and a width of $50 \mu \mathrm{m}$, the interpolated $\mathrm{S} / \mathrm{N}$ value is around 16.5. As expected, the $\mathrm{S} / \mathrm{N}$ is highest for the biggest pitch and the smallest width, $\mathrm{S} / \mathrm{N}=18$. The lowest value in the range is $\mathrm{S} / \mathrm{N}=13$.

For the inter-strip region the $\mathrm{S} / \mathrm{N}$ must be multiplied with the charge loss. For the $\mathrm{S} / \mathrm{N}$ value of the inter-strip region it is therefore a trade off between charge loss and increase of noise due to the increase of the total capacitance. In the right plot of Fig. 7 the S/N for the inter-strip region as a function of strip width and read-out pitch is shown. It can be seen that for the read-out pitch the relationship reverses compared to the on-strip case. The $\mathrm{S} / \mathrm{N}$ for the inter-strip region increases with decreasing read-out pitch. For the strip width, the total capacitance is still the dominating factor and the $\mathrm{S} / \mathrm{N}$ increases with decreasing strip width only. For wide pitches above $200 \mu \mathrm{m}$ this relation starts to reverse. The signal loss becomes dominating and the width of the strip has to be increased in order to increase the $\mathrm{S} / \mathrm{N}$ for the inter-strip region. For a read-out pitch of $200 \mu \mathrm{m}$ and a strip width of $50 \mu \mathrm{m}$ the $\mathrm{S} / \mathrm{N}$ ratio is about 12 . For the range investigated in the plots in Fig. 7 the maximum is at 13.5 and the minimum at 11. A possible charge collection deficiency due to the decrease in coupling capacitance with decreasing strip width can be neglected in the parameter space investigated here.

Based on these studies, the geometry of region A was chosen for the $7.8 \mathrm{~cm}$ wide and $11 \mathrm{~cm}$ long Inner Tracker modules incorporating 384 read-out strips. For the two-sensor ladders in the side boxes the thickness was increased to $410 \mu \mathrm{m}$ in order to ensure sufficient signal in the presence of the increased noise due
TABLE II

Performance Parameters. THE Numbers Behind the SONSOR TyPes CORRESPOND TO THE NuMBER OF SENSORS BONDED TOGETHER. THE TWO S/N VALUES SEPERATED by a SLASh REPRESENT the ON-StRIP VALUES AND THE INTER-STRIP VALUES

\begin{tabular}{|c|c|c|c|c|}
\hline Type & S/N & efficiency [\%] & noiserate [\%] & resolution $[\mu \mathrm{m}]$ \\
\hline LHCb 1 & $16.4 / 11.7$ & 99.7 & 0.04 & 41.58 \\
\hline LHCb 2 & $11.5 / 8.3$ & 99.4 & 0.05 & 47.52 \\
\hline LHCb 3 & $8.6 / 6.4$ & 96.6 & 0.06 & 53.46 \\
\hline CMS3 & $21.6 / 17.5$ & 99.9 & 0.05 & 43.92 \\
\hline CMS3+flex & $16.4 / 13.0$ & 99.8 & 0.06 & 49.41 \\
\hline
\end{tabular}

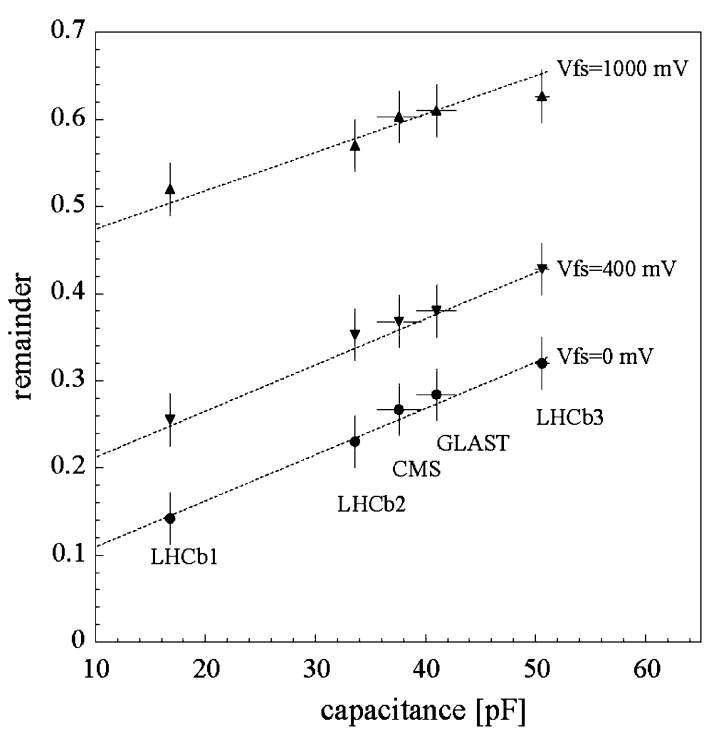

Fig. 8. Signal remainder after $25 \mathrm{~ns}$, i.e., one bunch crossing, as a function of the Beetle's shaping time $\left(V_{f s}\right)$.

to higher load capacitance. For the sensors used for the TT-station, the most cost efficient solution which fully matches the requirements, is to employ the tested sensor design from the outer barrel of the CMS Silicon Tracker [7]. The $500 \mu \mathrm{m}$ thick sensors have a width of $9.6 \mathrm{~cm}$ and a length of $9.4 \mathrm{~cm}$. The 512 read-out strips with a pitch of $183 \mu \mathrm{m}$ and a w/p of 0.25 are read out via 4 Beetle [8], [15] read-out chips.

In Table II the main performance parameters of the ladders as measured in the test beam are summarized. $\mathrm{S} / \mathrm{N}$ values are quoted for on-strip data and inter-strip data. The studies have shown that for a $\mathrm{S} / \mathrm{N}$ value of around 10 full cluster reconstruction efficiency is obtained. The shaping time was varied by means of the $V_{f s}$ setting in order to study the remaining signal $25 \mathrm{~ns}$ after the maximum, i.e., at the time of the consecutive bunch crossing. The signal width increases with increasing load capacitance as seen in Fig. 8. It is found that with $V_{f s}$ settings below $400 \mathrm{mV}$ the signal remainders can be kept below $30 \%$ and $50 \%$ as required for the Inner Tracker and the TT-station, respectively.

Measurements of the $\mathrm{S} / \mathrm{N}$ ratio for CMS-OB2 sensors, which were the thickest sensors under test, as a function of the bias voltage are shown in Fig. 9. Full charge collection efficiency is obtained at a bias voltage of about 300 Volt. Measurements of the backplane capacitance as a function of the bias voltage revealed a depletion voltage of around 200 Volt [10]. This shift in depletion voltage is due to the fast read-out and the charge collection time being at the same order of magnitude. The plateau 


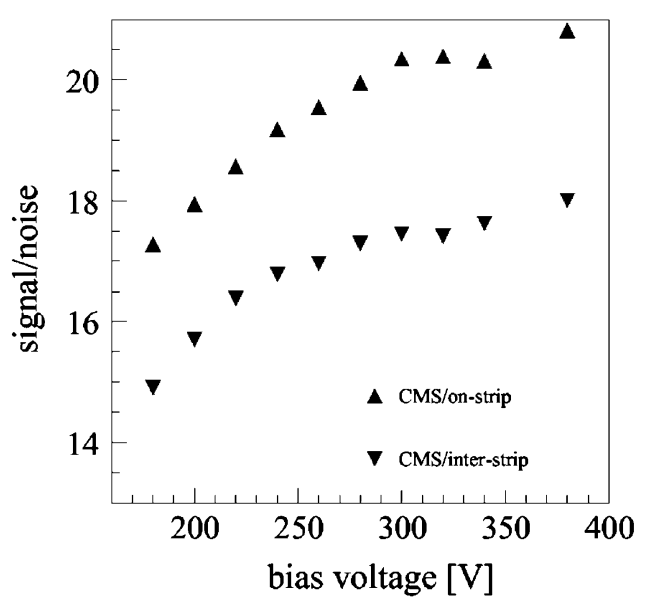

Fig. 9. The $\mathrm{S} / \mathrm{N}$ as a function of the bias voltage for the $500 \mu \mathrm{m}$ thick CMS-OB2 sensors. The upper points show the $\mathrm{S} / \mathrm{N}$ values for particles passing the region close to a read-out strip, whereas the lower points were obtained for particles in the inter-strip region.

of full charge collection efficiency is reached at around 1.5 times the depletion voltage. A factor of 1.5 up to 2 was derived for the various geometries of the prototype modules [12]. The difference in $\mathrm{S} / \mathrm{N}$ between inter-strip region and on-strip region stays constant for increasing bias voltage after the plateau is reached, showing that the charge loss in the inter-strip region cannot be decreased with higher over-depletion.

In a recent test-beam, a TT-module consisting of three CMS-OB2 sensors and a $40 \mathrm{~cm}$ Kapton interconnect cable was tested and it was shown that the signal-to-noise behaviour was as expected from the total load capacitance and the fit shown in Fig. 3 [13]. The performance of irradiated ladders was also studied in this test-beam at room temperature. LHCb multi-geometry prototype sensors were irradiated completely in a 24 $\mathrm{GeV} / \mathrm{c}$ proton beam at the CERN PS. For a one sensor module irradiated up to a dose of approximately $1.14 \times 10^{13} \mathrm{~cm}^{-2}$, full charge collection efficiency could still be obtained well below the specifications for the breakdown voltage of 500 Volt. The results show no significant charge loss in the irradiated sensor. Laboratory measurements with a laser were carried out and are reported in [14].

\section{Electrical Tests FOR Production}

Production of the Silicon Tracker is still ongoing. An efficient detection scheme of potential defects of the ladders, namely shorted channels, broken bonds and pinholes, is crucial for the quality control of the sensors. In order to study possible detection methods, defects were artificially introduced in a prototype module consisting of three $410 \mu \mathrm{m}$ thick sensors [12]. The DC-pad and AC-pad of one channel were bonded together in order to simulate punch throughs in the dielectric of the microstrips. Wire bonds were removed on different locations of the ladder and AC-pads of neighbouring channels were bonded together.

These defects cause a change in the load capacitance and thus affect the response of the Beetle read-out chip. Pulse shape scans were performed using the internally generated test pulses

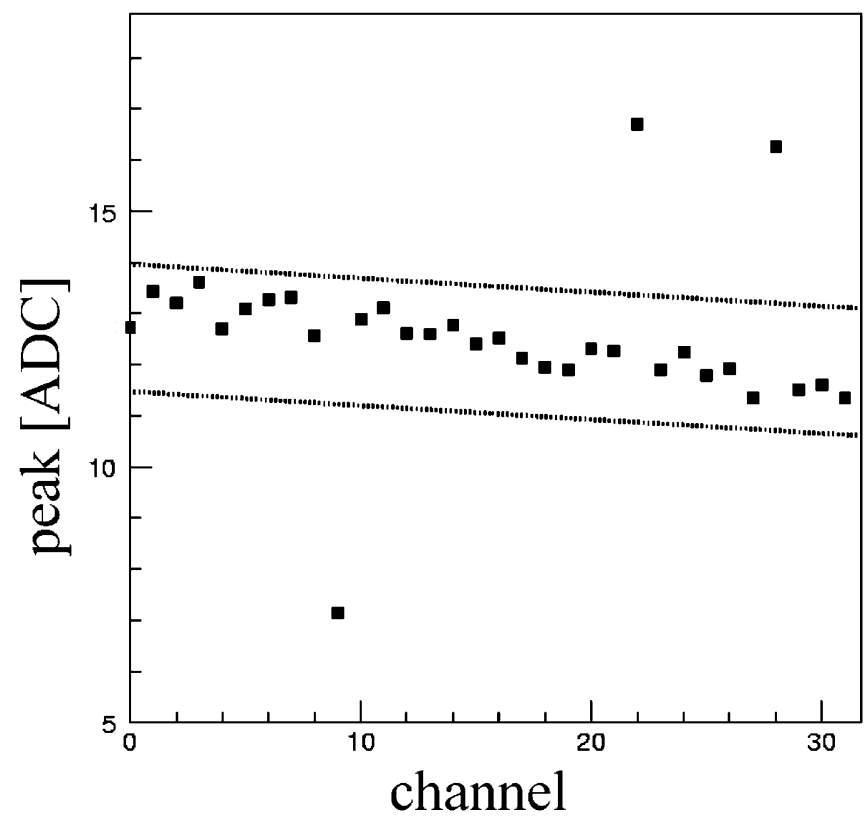

Fig. 10. Distribution of the peak heights of the internally generated test pulse. For two channels with a broken bond between middle and first sensor the pulse height is higher than the average. For a shorted channel the pulse height is smaller due to the increase of load capacitance. The dashed lines indicate three standard deviations after discarding the defects from a linear fit to all channels. Every fourth channel is plotted due to the fourfold test pulse pattern of the Beetle 1.2 chip.

of the Beetle, for fully depleted sensors. Defects were identified as channels whose pulse height was found to deviate by more than three standard deviations from the average of all channels, after discarding outliers and taking into account the linear slope. As shown in Fig. 10, a linear fit was used to allow for an overall linear trend over the read-out channels. Channels with broken bonds have less capacitance, which increases the gain in the amplifier. Shorted channels appear to have a lower peak height, due to the increase in capacitance. This makes it easy to distinguish between the two defects. Additional information can be gained from the width of the pulse shape. The distribution of the ratio of the signal remainder $25 \mathrm{~ns}$ after the peaking time to the peak height is shown in Fig. 11.

Punch-throughs in the dielectric permit leakage currents to flow into the pre-amplifier. For high leakage currents, as they occur after several years of irradiation, this could affect the performance of the pre-amplifier. Therefore it is desireable to detect pinholes already during production and to study if the corresponding read-out strips need to be disconnected from the pre-amplifier. While for fully depleted sensors, the artificially introduced pinholes did not show any significant noise pattern or any other deviations in the pulse shapes, the effect of the pinholes could be seen when a small forward bias of -0.5 Volt was applied. To protect the detector from any potential damage the current source was limited. The observed output signal of the Beetle chip for channels with pinholes was almost zero, as is shown in the left plot of Fig. 12. In the right plot of Fig. 12 the response of a pinhole to the test pulses is shown when no bias voltage was applied. The pinhole results in a significant decrease in peak height as well as a significant undershoot. Furthermore the channels around the pinhole seem to be slightly deteriorated. 


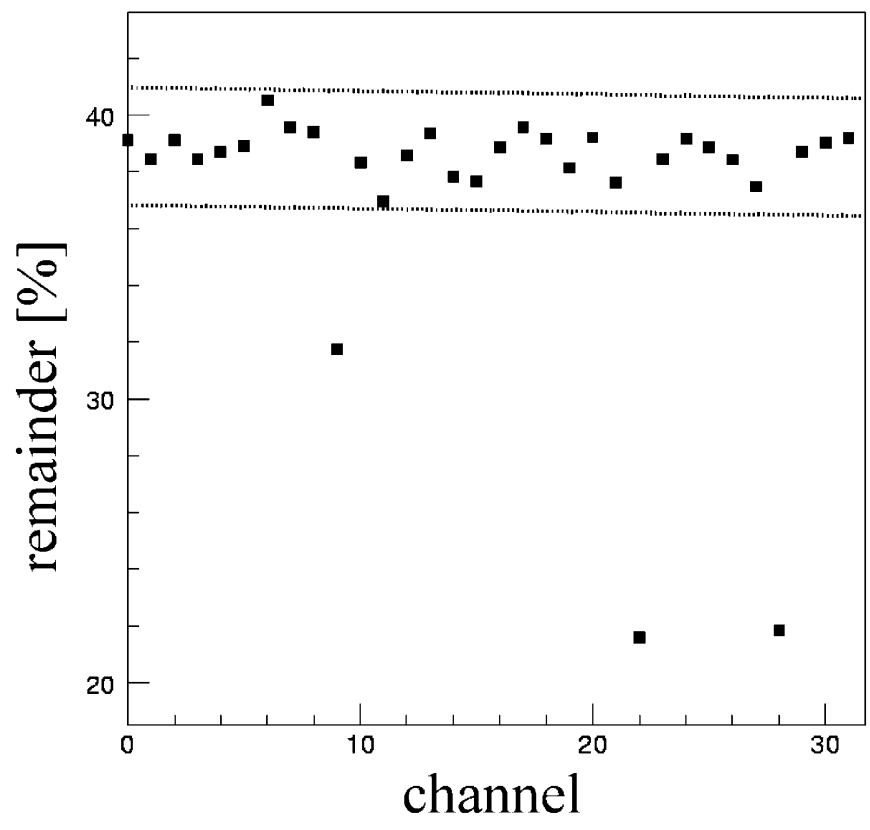

Fig. 11. Distribution of the signal remainder, $25 \mathrm{~ns}$ after the peaking time. Shorts and broken bonds appear as having a smaller remainder than the average. The dashed lines indicate three standard deviations after discarding the defects from a linear fit to all channels. Due to the particular test-pulse pattern, only every fourth channel is plotted.
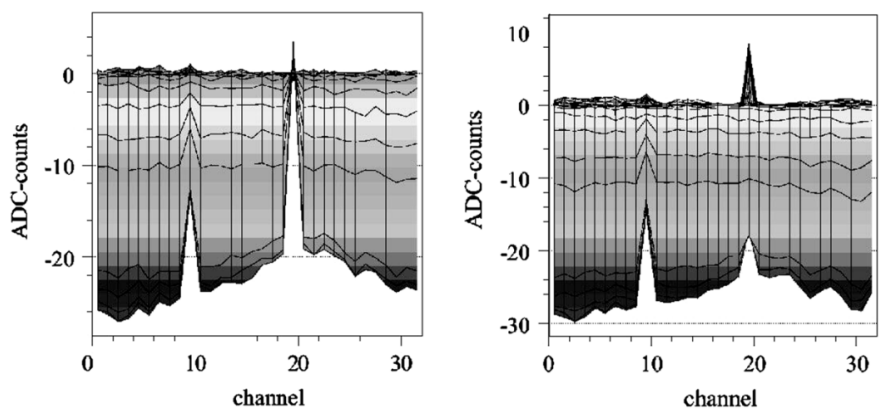

Fig. 12. Projection of the pulse shapes. On channel 9 a shorted channel is identified. The artificially indtroduced pinhole on channel 19 shows almost no response for a slightly ( -0.5 Volt) forward biased silicon sensor, as shown in the plot on the left. No bias voltage was applied for the plot on the right. The relative change of the shorted channel is unchanged. The pinhole shows significantly less response to the test pulse and deteriorates neighbouring channels. The polarity of the test pulses was negative. Due to the particular test pulse pattern, only every fourth channel is plotted.

\section{CONCLUSION}

The LHCb Silicon Tracker uses silicon strip detectors with up to $38 \mathrm{~cm}$ long read-out strips, a large pitch of $\approx 200 \mu \mathrm{m}$ and fast read-out electronics adapted to the $40 \mathrm{MHz}$ bunch crossing rate at the LHC. Test-beam measurements of the prototype modules have shown a performance according to expectations and within the specifications of the LHCb experiment. The internal test pulse generator of the Beetle pre-amplifier chip can be utilised to detect defects during the production. Laboratory measurements have demonstrated that shorts, broken wire bonds and pinholes can be detected at high significance.

\section{REFERENCES}

[1] LHCb Reoptimized Detector, Design and Performance, CERN/LHCC 2003-030, LHCb Collaboration.

[2] LHCb VELO, CERN/LHCC 2001-011, LHCb Collaboration.

[3] LHCb Inner Tracker, CERN/LHCC 2002-029, LHCb Collaboration.

[4] Lhcb Outer Tracker, CERN/LHCC 2001-024, LHCb Collaboration.

[5] F. Lehner, Description and Evaluation of Multi-Geometry Silicon Prototype Sensors for the LHCb Inner Tracker, LHCb note 2002-038.

[6] [Online]. Available: http://scipp.ucsc.edu/groups/glast/detector, GLAST Collaboration

[7] J.-L. Agram, The Silicon Sensors for the Compact Muon Solenoid Tracker-Design and Qualification Prodedure, CMS note 2003-015.

[8] U. Trunk, CERN/LHCC 2003-055.

[9] M. Agari, Testbeam Measurements on Prototype Ladders for the $\mathrm{LHCb}$ TT Station and Inner Tracker, LHCb note 2003-082.

[10] J. Gassner, Capacitance Measurements on Silicon Micro-Stip Detectors for the TT Station of the LHCb Experiment, LHCb note 2003-081.

[11] S. Braibant, "Investigation of Design Parameters and Choice of Substrate Resistivity and Crystal Orientation for the CMS Silicon Microstrip Detector," Nucl. Instrum. Methods Phys. Res. A, vol. A426, pp. 87-93, 1999.

[12] S. Köstner, "Large area silicon tracking detectors with fast signal readout for LHC experiments," Ph.D. dissertation, Technical Univ., Vienna, Switzerland, 2005.

[13] M. Agari, Measurements of a Prototype Ladder for the TT Station in a $120 \mathrm{GeV} / \mathrm{c} \pi^{-}$Beam, LHCb note 2004-103.

[14] C. Lois, Laboratory Measurements on Irradiated Prototype Ladders for the LHCb Inner Tracker, LHCb note 2004-112.

[15] U. Trunk, "Performance of the beetle readout chip for LHCb," in Proc. 9th Workshop Electronics for the LHC Experiments, Amsterdam, The Netherlands, Sep. 29-Oct. 3, 2003, pp. 443-447. 